FRANK MEHRING

\title{
Dissens in der Emigration: Der deutsch-amerikanische Abolitionist Karl Follen
}

\begin{abstract}
This paper analyzes the role of Karl Follen in the New England abolitionist movement. Despite his efforts to overcome his reputation as a German radical political activist in exile, his protest against slavery has been continually linked to the assassination of August von Kotzebue by Karl Sand, one of Follen's student followers. While biographers have focused on the continuity in Follen's transatlantic search for freedom, this paper emphasizes the differences between the cultural environment in Germany and the United States, which ultimately determined the nature of Follen's dissent. Working as a professor at Harvard University, Follen became an American citizen and adapted his ideas of destiny, promise and rhetorical prophecy to his new socio-political surroundings. Although he promoted revolution and even assassination during the German wars of liberation (1813-15), Follen turned into an Emersonian reformer advocating self-reliance, the abolition of slavery and the emancipation of women after his escape to America. In order to understand the dynamics of Follen's dissent and non-violent approach regarding social reforms, the rhetoric of William Lloyd Garrison serves as a foil. While Follen desperately tried to become a representative American, his uncompromising advocacy for human rights based on the Declaration of Independence ultimately estranged him from many nativists who denounced him as a dangerous "foreign meddler." It appeared as if German notions of freedom were incompatible with the American concept of democracy. This is why Follen's controversial activities, his cultural contributions and their far-reaching repercussions need to be evaluated in the broader context of democratic vistas in German-American discourses.
\end{abstract}

\begin{abstract}
We, the humble and devoted advocates of the oppressed, address you, our fellow-citizens, in behalf of more than two millions of men, our countrymen, whom we, the people of these United States, have doomed to absolute and perpetual bondage.
\end{abstract}

Karl Follen

\section{Einleitung}

Karl Follen war nicht nur ein Pionier der transatlantic encounters, wie Ursula Brumm in Bezug auf den Deutsch-Amerikaner erklärt (Brumm 1995, 146), sondern auch eine Ausnahmeerscheinung in der Auseinandersetzung mit der amerikanischen Kultur im 19. Jahrhundert. Denn im Gegensatz zur verbreiteten Vorstellung, dass sich die Mehrzahl der deutschen Immigranten mit den gegebenen 
sozioökonomischen Strukturen arrangierte und vielfältige Beiträge für die fortschrittliche Entwicklung in den Vereinigten Staaten leistete, zeigt das Beispiel Karl Follen ein differenzierteres Bild. ${ }^{1}$ Sein unumstößlicher Wille, sich als vorbildlicher amerikanischer Republikaner zu akkulturieren, veranlasste ihn dazu, in den 1830er Jahren für die Sklavenbefreiung und die Frauenemanzipation politisch aktiv zu werden. Der Widerspruch zwischen den in der Declaration of Independence verankerten Menschenrechten und der Realität der Sklaverei begründete seinen Protest gegen die aus seiner Sicht beklagenswerte Verletzung amerikanischer Werte.

Europäische Reisebeschreibungen und Briefe von Immigranten lieferten die ersten kritischen Bewertungen der amerikanischen Demokratie, indem sie deren Tragfähigkeit als Gegenentwurf zum europäischen Feudalsystem erörterten. Alexis de Tocquevilles Democracy in America avancierte zu einem der meistbefragten Texte, wenn es darum ging, Auskünfte über die Wechselwirkungen zwischen Demokratie und Kultur im Amerika des 19. Jahrhunderts zu erhalten (Mitchell 2002, 4). Mit scharfem Blick analysierte der französische Intellektuelle 1831 u.a. die gesellschaftspolitische Situation und den Umgang mit der Sklaverei. Zum Zeitpunkt als Tocqueville die Vereinigten Staaten bereiste, begann auch Follen sich zunehmend kritisch mit den in der Unabhängigkeitserklärung begründeten unveräußerlichen Rechten und der Legitimation der Sklaverei auseinander zu setzen. Im Unterschied zur Amerikaerfahrung in der deutschen Reise-, Tagebuch- und Abenteuerliteratur eines Gottfried Duden, Friedrich Gerstäcker, Charles Sealsfield, Ludwig de Wette oder Prinz Maximilian zu Wied beschäftigte sich Follen jedoch intensiv mit rechtlichen Belangen der schwarzen Bevölkerungsgruppen in Amerika. Während die deutsch-amerikanische Reiseliteratur die im frühen 19. Jahrhundert vorherrschende Stereotypisierung der "schwarzen Bevölkerung" als "primitive Wilde" aufgriff und fortschrieb (Sammons 1998, 152), setzte Follen neue Akzente. Er instrumentalisierte seine juristischen Fachkenntnisse und charismatische Rhetorik, um sich aktiv für eine Umgestaltung der Lebensbedingungen der Afroamerikaner nicht nur in den Südstaaten, sondern auch im liberal eingestellten Norden einzusetzen. Er agierte dabei nicht als kultureller Außenseiter oder touristischer Beobachter, sondern als amerikanischer Staatsbürger. So entstanden Konfliktsituationen, die über die Presse mit dem Hinweis auf seine Vergangenheit verschärft wurden. Die deutsch-amerikanische Identität Follens bildete in der Öffentlichkeit einen zentralen Ansatzpunkt, um seine politischen Aktivitäten und Publikationen kritisch zu bewerten. Sein Protest gegen

1 Don Heinrich Tolzmanns positivistischer Geschichtsüberblick The German-American Experience (2000) steht in der Tradition von Publikationen, deren Zentrum die Aufwertung deutsch-amerikanischer Traditionen bildet; dazu gehören vor allem Faust (1927) und Pochmann (1957). Kritischere Rückfragen an das Konfliktpotential deutsch-amerikanischer Begegnungen stellen Trommler und Shore (2001). Fluck und Sollors (2002) gehen der Frage nach, welchen Gewinn eine akademische Beschäftigung mit dem bis dato vernachlässigten Quellenreichtum von mehr als dreihundert Jahren deutsch-amerikanischer Literaturproduktion zu Tage fördern kann. Bezeichnenderweise fehlen in beiden Überblicksdarstellungen Hinweise auf die Rolle von Karl Follen als Vermittler deutschen Gedankenguts. 
die Sklaverei galt vielen Zeitgenossen als unwillkommene Einmischung in inneramerikanische Angelegenheiten. Follens Freiheitskampf im Deutschland des Vormärz und sein Engagement für die Rechte der Afroamerikaner wirft noch heute zahlreiche Fragen auf, die Aufschluss über Friktionspunkte in der deutsch-amerikanischen Begegnung geben können. In welchem Verhältnis stehen Follens intellektuelle Prägungen im Deutschland des Vormärz mit seinen Reformbemühungen im Amerika der Jacksonian Era? Welche Transformationen durchlaufen seine Ideale im Prozess der Akkulturation in den Vereinigten Staaten? Welche Mechanismen der öffentlichen Wahrnehmung entstehen in Konfliktsituationen der deutsch-amerikanischen Begegnung?

\section{Vom deutschen Befreiungskrieger zum amerikanischen Reformer}

Karl Theodor Christian Follen kam am 4. September 1796 im oberhessischen Romrod im Hause seiner Großeltern zur Welt. Die in Giessen ansässige Familie war kurzfristig aus der Universitätsstadt vor den anrückenden napoleonischen Heeren aufs Land geflüchtet (Follen 1842, I, 3). Während seines Theologie- und Jurastudiums an der Ludoviciana (der heutigen Justus-Liebig-Universität Giessen) zwischen 1813 und 1818 entwickelte sich der glühende Anhänger der Ideen Friedrich Ludwig Jahns, Ernst Moritz Arndts und Johann Gottlieb Fichtes zu einem kämpferischen Gegner der französischen Vorherrschaft (Wüst 1936, 22). Als Gründer der radikalen Burschenschaftsvereinigung "Die Giessener Schwarzen" trug er massgeblich dazu bei, dass die Ludoviciana zu einem "Paradebeispiel der politisierten Vormärz-Universität" avancierte (Moraw 1990, 114). In seinen Gedichten beschwor Follen das politische Attentat als legitimes Mittel, um einen ungerechten Obrigkeitsstaat zu beseitigen und die Ideale der französischen Revolution in einem deutschen Bundesstaat zu verwirklichen. 1818 übernahm Follen eine Dozentur an der juristischen Fakultät der Universität Jena, die er bereits ein Jahr später wegen des Vorwurfs der Mitwisserschaft im Mordfall August von Kotzebues zugunsten einer vierjährigen Lehrtätigkeit an der Baseler Universität im schweizerischen Exil aufgeben musste. Die staatlichen Kontrollmechanismen der Karlsbader Beschlüsse und die strafrechtliche Verfolgung zwangen Follen schliesslich zur Flucht in die USA. Sein Kontakt zum Marquis de Lafayette, dem französischen Unterstützer der amerikanischen Unabhängigkeit, ermöglichte ihm bei seiner Ankunft in New York im Dezember 1824, Freundschaft mit George Ticknor und John Thornton Kirkland zu schließen. Beide Harvard-Professoren gehörten zu einer Gruppe von aufgeschlossenen Intellektuellen, die sich zu diesem Zeitpunkt für die Verbreitung deutschen Bildungsgutes im amerikanischen Geistesleben einsetzten (Totten 1964, 68). Nach seiner Ankunft in Neuengland amerikanisierte Follen seinen Vornamen und arbeitete zunächst als Deutschlehrer. Sein vielbeachtetes Lesebuch A German Reader for Beginners [by Charles Follen] (1826) und die Grammatik A Practical Grammar of the German Language (1828) lieferten wichtige Grundlagen für die Diskussion über deutsche Literatur unter den amerikanischen Transzendentalisten (Pochmann 1957, 116). Sie verhalfen ihm zu 
rascher Anerkennung in Boston. Der gesellschaftliche Aufstieg führte von der Lehrtätigkeit über die Heirat mit Eliza Lee Cabot (1787-1860) zur Professur für deutsche Literatur an der Harvard University (Follen 1842, I, 261-4). Anhand der von ihm verfassten Grammatiken und Lesebücher vermittelte er den jungen Intellektuellen von Cambridge und Boston Einblicke in die deutsche Literaturgeschichte. Seine enthusiastischen Vorträge über Schiller und seine kritische Auseinandersetzung mit dem künstlerischen Werk Goethes schätzten vor allem einflussreiche Persönlichkeiten wie Ralph Waldo Emerson und Theodore Parker. Die durch Follens Position an der Harvard University verstärkte Rezeption der deutschen Klassik lässt sich auch an Publikationen von Schriftstellern wie Bronson Alcott, Margaret Fuller, Frederic Hedge, George Ripley und Henry David Thoreau nachweisen (Mehring 2004, 185-9).

Seit 1831 setzte sich Follen aktiv für die Verwirklichung der Menschenrechte ein, indem er sich der brisanten Frage der Sklaverei und rechtlichen Gleichstellung der Frauen annahm. Sein energisches Engagement führte zur Zusammenarbeit mit dem kämpferischen Abolitionisten William Lloyd Garrison (Spindler 1917, 189). Die ethnischen Assimilationskräfte der offenen amerikanischen Gesellschaft begannen jedoch rasch brüchig zu werden, als sich der eingebürgerte Immigrant in abolitionistischen Kreisen engagierte, um die Menschenrechte auch für Afroamerikaner einzufordern. Damit verließ Follen seine auf Assimilation ausgerichtete Lebensführung und trat selbstbewusst für gesellschaftspolitische Reformen ein. Die Phase der kritischen Auseinandersetzung mit der Sklavenfrage fiel mit der erfolgreichen Umsetzung beruflicher Ambitionen als (erstem) Professor für deutsche Literatur an der Harvard University zusammen. Die politische Intervention als Vizepräsident der Massachusetts Anti-Slavery Society bedingte eine neue Rollenzuweisung in der Öffentlichkeit. Seine deutsche Vergangenheit als radikaler Burschenschaftler und sein Status als unbequemer Einwanderer führten zu Konflikten in der deutsch-amerikanischen Begegnung.

Die auf beiden Seiten des Atlantiks neuerdings einsetzende Aufarbeitung von Follens Leben und Wirken verläuft sehr unterschiedlich. In Deutschland haben sich vor allem Historiker mit der Frage der Mitschuld am Mord an August von Kotzebue durch Karl Sand beschäftigt. Maßgeblich für die Bewertung seiner Persönlichkeit war die von Heinrich von Treitschke 1882 vorgegebene Interpretationsrichtung:

Ein Fanatiker des harten Verstandes, im Grunde ein unfruchtbarer Kopf, aber von seltenem dialektischem Scharfsinn, ein frühreifer, ganz mit sich einiger Charakter, der nach der Weise radikaler Propheten sich den Anschein dämonischer Unergründlichkeit zu geben wusste und manchen seiner jungen Genossen wie der Alte vom Berge vorkam. (Treitschke 1927, 438)

Treitschkes Negativzeichnung von Follen als radikalem Brandstifter wirkt bis heute nach. Wolfgang Hardtwig kritisiert die antimodernen Züge in Follens Wesen und gibt zu bedenken, dass sich hier ein "neuartiges totalitäres Denken unter dem Vorzeichen des revolutionären Nationalismus" Bahn brach (Hardtwig 1985, 14). Eine weniger polemisch überzeichnete Beurteilung als Treitschke legten 
Hermann Haupt 1907 mit einer biographischen Aufarbeitung von Follens Wirken als Burschenschaftler und Julia Wüst 1936 mit einer ideengeschichtlichen Untersuchung vor. Bedauerlicherweise gehen beide Arbeiten kaum auf die zweite Lebenshälfte Follens in Amerika ein. Die amerikanische Deutung stellt die isolationistischen Folgen seines politischen Engagements, aber auch die vielfachen Verdienste als Vermittler deutschen Gedankenguts in den Vordergrund. Als erste bemühte sich Eliza Lee Cabot Follen bereits kurz nach dem Tod ihres Mannes 1841 um eine Gesamtschau von Follens Leben. In diesem fünfbändigen Werk, bestehend aus Memoiren, Briefen, Vorlesungen, Reden und Pamphleten, bleibt weder die deutsche noch die amerikanische Wirkungsgeschichte unberücksichtigt. Nachfolgende amerikanische Publikationen zielten darauf ab, eine Kontinuität zwischen Follens radikalem Wirken als deutschem Burschenschaftler und amerikanischem Abolitionisten aufzuzeigen. Die Rezeption in den Vereinigten Staaten gestaltete sich damit zwar ausgewogener als die chronologisch verkürzte Betrachtung in Deutschland, die Abhandlungen erliegen jedoch der Gefahr, Follens Leben auf einen ununterbrochenen Kampf für die Freiheit in radikalen Zirkeln zu reduzieren, ohne die Wandlungen seiner Identitätssuche gebührend zu thematisieren.

Der Deutsch-Amerikaner Albert Bernhardt Faust feiert Follens Ankunft in Amerika als Bereicherung für das intellektuelle Leben Neuenglands. Nur sein früher Tod habe einem größeren Ansehen entgegengewirkt (Faust 1927, I, 427). Faust sieht in Follens Einsatz für die Freiheit eine schicksalhafte Vorbestimmung: "But the man who had become an exile for the sake of liberty in Germany was destined to play a part also in the struggle for the liberty of mankind" (Faust 1927, I, 216). George Washington Spindler konzentriert sich in seiner Biographie auf Follens Wirken in Amerika, ohne allerdings die deutsche Vergangenheit auszublenden. Nichtsdestotrotz bleibt die Zweiteilung seines Werkes in "Follen in Europe" und "Follen in America" unausgeglichen. Dem fünfjährigen Exil schenkt er lediglich kursorische Beachtung. Spindler argumentiert dementsprechend: "It will be seen that Follen as a product of the classical period of German literature and philosophy assimilated even in his youth the spiritual forces of his time, and that these not only determined his activity in Europe but also that of his American career" (Spindler 1917, 9). Während die Mehrzahl an Publikationen von Gustav Philipp Körners Das deutsche Element in den Vereinigten Staaten 1818-1848 von 1880 bis zu Henry Pochmanns monumentaler Gesamtschau A German Culture in America: Philosophical and Literary Influences, 1600-1900 von 1957 die Verdienste Follens in den Vordergrund rückten, nimmt Edmund Spevack, Follens jüngster Biograph, eine andere Haltung ein. Er betont die Determiniertheit von Follens Aktivitäten in Amerika durch seine Erfahrungen mit der deutschen Literatur und Philosophie des Vormärz. Spevack verurteilt Follens politisches Engagement in den USA, indem er eine bruchlose Übertragung der von Gewalt inspirierten revolutionären Ideen des Vormärz auf den Abolitionismus impliziert: "As always, his sense of moral duty and of absolute commitment to a cause which he believed to be just and true came before anything else" (Spevack 1997, 206; Hervorhebung FM). Indem Spevack das Augenmerk auf die Frage der Gewaltanwendung zur Durchsetzung freiheitlicher 
Prinzipien in Deutschland und Amerika richtet, isoliert er Follen als gefährlichen Dissidenten und verkennt dessen vielfältige Neuakzentuierungen im demokratischen Umfeld der Neuen Welt.

Die deutsch-amerikanischen Spannungen bringen methodologische Probleme mit sich. Eine erneute Auseinandersetzung mit dem Leben und Werk Karl Follens darf sich nicht damit begnügen, von der Forschung vergessene Texte zu entstauben. Vielmehr muss es im Sinne von Winfried Fluck zu einer Kontextualisierung und zu Vergleichen kommen, um die Ideen in einem weiteren kulturellen und intellektuellen Feld zu verankern (Fluck 2002, 176). Im folgenden soll Follen nicht nur in den kulturellen Kontext der neuenglischen Abolitionismusbewegung eingeordnet werden, es sollen auch Parallelen und Unterschiede zu anderen Emigranten aufgezeigt werden. So lassen sich nicht nur die Kontinuitäten sondern auch die Wandlungen im Denken von Follen erkennen.

\section{Der Abolitionismus als Wendepunkt in Follens Entwicklungsprozess}

In deutschen Zeitschriften vor 1830 fand im Gegensatz zum europäischen und transatlantischen Ausland die Diskussion der Menschenrechtsdebatte in Verbindung mit der Sklavenfrage kaum statt. Die Brisanz dieses Topos "blieb auf publizistischer Ebene verborgen” (Depkat 1998, 371). Follens Erwartungen prägten die aus Reiseberichten und dem Studium der Unabhängigkeitserklärung vermittelten Ideale von Freiheit und Gleichheit. Seit 1817 diskutierte er mit seinem älteren Bruder August Follen die Möglichkeit, einen Staat mit deutschen Siedlern im Westen Amerikas zu gründen, der nach eigenen freiheitlichen Idealen organisiert wäre. Eine dort aufzubauende Universität sollte Ausgangspunkt deutscher Bestrebungen sein, einen Freistaat zu errichten. "Auf diese Weise kann es gelingen, die Deutschen in Nordamerika zu Einem, auf dem Kongress sich vertretenden Staate [...] zu verbinden, welcher ein Vorbild für das Mutterland und in vielfältiger Beziehung für seine Befreiung werden kann.”2 Obwohl sein jüngerer Bruder Paul und Friedrich Münch die Idee eines deutschen Modellstaates in der eigens gegründeten Giessener Gesellschaft weiter verfolgten (Heubner 1962, 62), änderte Follen seine Einstellung schon bald nach der Ankunft in der amerikanischen Realität. Zwar bliebt sein ideeller Auftrag, ein freiheitliches Staatsideal in den Vereinigten Staaten umzusetzen bzw. zu wahren, weiterhin bestimmend. Die utopische Idee, mit Hilfe eines elitären Personenkreises den Umsturz der Aristokratie von einem außereuropäischen Land einzuleiten, um eine deutsche Republik zu erstreiten, ließ er jedoch fallen.

Follen suchte in den Vereinigten Staaten vor allem eines: die Verwirklichung des Versprechens nach individueller Freiheit und die Einhaltung der in der Ver-

2 Der Auszug basiert angeblich auf Karl Follens unbetitelter Denkschrift von 1819. Beilage CC des Hauptberichts vom 14.12.1827. Beigefügt in BA Frankfurt, DB 7/11, Fol. 121-130. Fol. 129. Die Urheberschaft ist allerdings umstritten. Follen setzte sich mit den Möglichkeiten eines solchen Modellstaates auseinander. Sein Bruder Paul nahm diese Konzeption später wieder auf. Vgl. Luys, 1992, 174-6. 
fassung verankerten Menschenrechte. Auf der Überfahrt nach New York brachte er seine Erwartungen in einem Gedicht auf den Punkt: "Dort an des Meeres anderm Strand, / Dort ist der Freiheit, dort der Menschheit Vaterland" (Follen 1842, I, 128). Das verklärte Amerikabild resultiert aus dem Pessimismus der napoleonischen Kriege und der Restaurationszeit: Amerika galt als erfolgversprechendes Auswanderungsland, als Land der unberührten Natur und als demokratische Republik. Follens Wahrnehmung blieb auch während der ersten Jahre ungetrübt. So tauchte beispielsweise die Diskriminierung der Afroamerikaner vor 1830 nicht in seinen Überlegungen auf.

Den Ausgangspunkt für die aktive Mitgestaltung der abolitionistischen Forderungen liefert eine Anekdote von Follens Frau, welche die Fachwelt unkritisch als hinreichende Erklärung für Follens Engagement übernimmt. Die zufällige Begegnung mit einem älteren ehemaligen Sklaven im Jahr 1831, also sieben Jahre nach Follens Ankunft in den USA, habe den Charakter einer Offenbarung besessen. Er wies Follen auf die Antisklavenbewegung hin und machte ihn auf das Pamphlet des schwarzen Abolitionisten David Walker aufmerksam, das in Boston im Herbst 1829 erschienen war. "Walker's Appeal" zeichnet sich durch eine aggressive, umstürzlerische und kompromisslose Rhetorik aus, die in der Sekundärliteratur mit Follens agitatorischen Zeilen wie "Schlagt eure Plager todt, / Rettet das Land!" aus "Das Große Lied" rückgekoppelt wird (Follen 1842, I, 588). Die Vergleiche scheinen offenkundig: Wie in Deutschland nach dem Wiener Kongress geht es auch in den Vereinigten Staaten um eine Gruppe von Unterdrückten, die unter obrigkeitsstaatlicher Macht leiden. Obwohl der Anteil der afroamerikanischen Bevölkerung in Massachusetts bei nur einem Prozent lag (Tocqueville 2004, 339), ist es unwahrscheinlich, dass Follen nicht schon früher über die rechtliche Situation der schwarzen Bevölkerungsgruppen informiert war. Die Gründe für sein ungewöhnlich apolitisches Leben bis 1831 und die nachfolgende Identifizierung mit der kämpferischen Attitüde Garrisons müssen daher tiefer liegen. Im folgenden gilt es, der neuen Selbstpositionierung Follens und der veränderten Wahrnehmung seiner Vergangenheit in der Öffentlichkeit nachzuspüren.

Eine Untersuchung der Schriften und Reden Follens zeigt, inwiefern er eine eigenständige Position entwickelte. In Abgrenzung zum Stil und Inhalt der charismatischen Führungsperson William Lloyd Garrison geben sie Auskunft über die Neupositionierung des Deutsch-Amerikaners im intellektuellen Umfeld Neuenglands. Aus der Perspektive Follens stellte sich Garrisons kompromisslose Rhetorik als problematisch dar. Die Gegenüberstellung der beiden Denkansätze offenbart eine klare Veränderung in Follens Einstellung zur Frage der politischen Radikalität. Deshalb gilt es auch rückzufragen, ob die Schlussfolgerungen, die Biographen und zeitgenössische Anhänger in den Burschenschaftszirkeln aus den Ideen Follens zogen, nicht zu vorschnell waren.

In der Verurteilung der Sklaverei fallen zunächst zahlreiche Gemeinsamkeiten ins Auge, die Follens und Garrisons Kampf bis 1840 kennzeichneten. So betonten beide die christliche und philosophische Perspektive als Basis ihrer Argumentation 
(Garrison 1852, 48). Bereits in dem ersten Grundsatzprogramm, in dem wesentliche Prinzipien der Anti-Slavery Societies festgehalten wurden, erörtert Garrison die Notwendigkeit, in möglichst vielen Städten Gesellschaften zu gründen. Follen folgte dieser Anregung und gründete 1834 die Cambridge Anti-Slavery Society. Bezeichnenderweise gehörte zu den Zielsetzungen dieses Ablegers der Massachusetts Anti-Slavery Society, die Radikalität und Aggressivität jenes Redestils abzumildern, den insbesondere Garrison pflegte (Stange 1977, 31). Follens Einschätzung, dass mit der Emanzipation der Afroamerikaner die Emanzipation der Frauen unabdingbar einhergehe, teilte Garrison ebenso wie später Frederick Douglass. Was Garrison in "American Colorphobia” 1847 in seiner Forderung nach der politischen, sozialen und anthropologischen Gleichstellung der Afroamerikaner mit den sogenannten "northern whites" auf den Punkt brachte, manövrierte ihn auch in der Abolitionismusbewegung in den extremen Randbereich. Selbst Vertreter in den eigenen Reihen wie Gerrit Smith, Wendell Phillips, Frederick Douglass und Lydia Maria Child mochten ihm nicht immer in seiner radikalen Haltung folgen. Garrisons trotziger "Byronismus" (Mayer 1998, 209) und seine Abscheu gegenüber der an Konventionen orientierten Gesellschaft stehen in krassem Widerspruch zu Follens umsichtiger, vorsichtig kalkulierender Haltung in der Menschenrechtsdebatte.

Garrisons Pochen auf eine unverzügliche, unbeschränkte und kompensationsfreie Abschaffung der Sklaverei, das seine Schriften und Reden von seinem 24. Lebensjahr bis in den Bürgerkrieg hinein durchzieht, verdeutlicht die eigenständige Position Follens.

1. Garrison lehnte die Verfassung der Vereinigten Staaten rigoros ab, da er in ihr die Legalisierung der Sklaverei verankert sah. Der Inhalt verletze zentrale Prinzipen, die in der Amerikanischen Revolution erstritten wurden.

2. Garrison sprach sich ausdrücklich gegen die Kolonisationsbewegung aus und monierte das schleichende Fortschreiten der Sklaverei, das durch die wirkungslosen Versuche einer moralischen Überzeugungsarbeit verursacht würde. Stattdessen müsse es zu politischen Aufständen und der Polarisierung der öffentlichen Debatte kommen.

3. Garrison gehörte zu den frühen "Sezessionisten," die den Bruch der Vereinigten Staaten in einen sklavenfreien Norden und ausbeuterischen Süden in Kauf nahmen bzw. propagierten. Die Abschaffung der Sklaverei lasse sich nicht gewaltfrei verwirklichen. Bereits 1829 zeigte er sich überzeugt von einem unvermeidbaren Zusammenstoß unvereinbarer Interessen: "No; we must expect a collision, full of sharp asperities and bitterness" (Garrison 2000, 100).

Gerade der Mangel an Bereitschaft zur Gewaltanwendung, um freiheitliche Rechte durchzusetzen, unterscheidet Follen vom Herausgeber des Liberator. Im Gegensatz zu seiner Führungsrolle in der Burschenschaftsbewegung agiert Follen nun innerhalb eines demokratisch-republikanischen Systems, das er in Deutschland vergeblich zu etablieren suchte. Vor diesem Hintergrund wird verständlich, dass seine Argumentation auf die Nutzung des legalen Instrumentariums und eine Neuinterpretation der Verfassung setzte. Follen trat in Amerika zu keinem Zeit- 
punkt für eine Lösung des Konflikts mit Mitteln der Gewalt ein. Vielmehr beschwor er das Beschreiten rechtlicher Wege und die Notwendigkeit moralischer Aufbauarbeit. In einer Rede vor der Massachusetts Anti-Slavery Society 1836 erklärte er unmißverständlich seine Ablehnung von Gewalt: "Our rights, our duties, with regard to the oppressed, require and authorize the use of all lawful and moral means, to accomplish the great object of deliverance" (Follen 1842, I, 632).

Als die erste New England Anti-Slavery Convention in Boston vom 27. bis 29. Mai 1834 stattfand, übernahm Follen die Position des Vorsitzenden. Seine hier gehaltene "Address to the People of the United States" forciert in klaren Worten die sofortige Abschaffung der Sklaverei, indem er die Frage aus politischer, moralischer und ökonomischer Sicht analysiert und kritisch bewertet. Follen hebt die demokratischen Prinzipien der Unabhängigkeitserklärung hervor und leitet davon jene Rechte ab, die bis dato Afroamerikanern vorenthalten wurden. Ihm ging es nicht um eine Spaltung des Landes oder die Änderung der Konstitution. Vielmehr propagierte er eine Neuinterpretation, die eine Überarbeitung oder gar eine Abschaffung erübrige. Als negatives Vergleichsbeispiel dient die europäische Tradition des Ständesystems, das in Amerika dank der Revolution zugunsten einer demokratischen Verfassung überwunden werden konnte. Gleichzeitig mahnt er, dass sich mit der Sklaverei eine Praxis eingeschlichen habe, die gerade die verhassten europäischen Traditionen wieder einführte.

In der Anrede seines Publikums stellt sich Follen in die Reihe der amerikanischen Bürger, indem er an das Einheitsgefühl der Abolitionisten appelliert: "we, the humble and devoted advocates of the oppressed" (Follen 1842, V, 189; Hervorhebung FM). Charakteristisch ist die vorbehaltlose Selbstbefragung, um schließlich Lösungen für die angesprochenen Probleme zu liefern. Er beginnt damit, die schwerwiegenden Vorwürfe seitens der Südstaaten zu erörtern, die in der AntiSlavery Society eine Agitation zum Krieg, eine Provokation zur Spaltung des Landes und eine gegenseitige Aufhetzung der amerikanischen Bürger wähnten. Solchen Befürchtungen wirkt Follen unverzüglich entgegen: "No, it is none of these" (Follen 1842, V, 190). Er kommt vielmehr anhand zahlreicher Beispiele zu dem Umkehrschluss. Gerade die Sanktion der Sklaverei beschwöre und forciere die Gefahr eines Aufstandes. Denn die Betonung der Unterschiede innerhalb eines Landes, in der allen Menschen gleiche Grundrechte zustehen, führe zur Destabilisierung des sozialen Klimas. "Slavery is the true and lasting source of insurrection; it is the avowed or secret cause of all the serious differences between the members of this Union. Those, therefore, who directly or indirectly strive to secure the existence of slavery in this country, are nourishing the seeds of a servile and civil war" (Follen 1842, V, 217). Follen beabsichtigt mit seiner Rede, das Ideal der Aufklärung zu verfolgen. Es geht ihm um die Darlegung der Natur- und Menschenrechte, um innerhalb eines freiheitlichen Amerikas Bruchstellen zu reparieren. Den Ausgangspunkt bilden die Feierlichkeiten des Unabhängigkeitstages und die dahinter stehenden Ideale, die auch Garrison nicht müde wird, in ihren Widersprüchen zu thematisieren. "Every Fourth of July is to us a day of exultation for 
what we have done, and a day of humiliation for what we have left undone" (Follen 1842, V, 190). Er nimmt damit eine Haltung vorweg, die später Frederick Douglass popularisierte. In seiner Rede "What to the Slave is the 4th of July?," die er 1852 bezeichnenderweise einen Tag nach den Feierlichkeiten hielt, prangert er die heuchlerischen Wahrnehmungsmechanismen der weissen amerikanischen Öffentlichkeit gegenüber ihren eigenen Idealen an. "This Fourth [of] July is yours, not mine. You may rejoice, I must mourn” (Douglass 1893, 104).

Follens Argumentation versucht seine Zuhörer auf rationaler Ebene von dem Unrecht der Sklaverei und der Notwendigkeit ihrer unverzüglichen Abschaffung ("immediate abolition") zu überzeugen. Er räumt rechtliche Probleme aus dem Weg, indem er die scheinbaren Gegensätze zwischen Konstitution und Unabhängigkeitserklärung auflöst. Die gegenwärtige Auslegung der Verfassung sanktioniere zwar rechtlich die Sklaverei, dies sei allerdings kein Grund, die Möglichkeiten einer Neuinterpretation ungenutzt zu lassen. Um Inkonsistenzen mit der Unabhängigkeitserklärung beseitigen zu können, wartet Follen mit einer juristischen Neuauslegung des 4. Artikels, Paragraph 3, Absatz 3 auf. Darin heißt es: "No person held to service or labor in one state, under the laws thereof, escaping into another, shall in consequence of any law or regulation therein, be discharged from such service or labor, but shall be delivered up on claim of the party to whom such service or labor may be due" (Follen 1842, V, 208). Eine Harmonisierung der unveräußerlichen Rechte jedes Menschen mit der Unabhängigkeitserklärung sei dann erreicht, wenn der Begriff des Dienstverhältnisses nicht auf die Sklaverei übertragen werde. Die wichtigen rechtlichen Fortschritte, die für die Sicherung der freien Entfaltung des Individuums in die Unabhängigkeitserklärung Eingang fanden, dürften nicht durch einen einzigen Satz in der Verfassung ad absurdum geführt werden. Follen stellt klar, dass der Auftrag und das Ansinnen der Anti-SlaverySociety ausschließlich darin bestehe, vom Recht der freien Meinungsäußerung Gebrauch zu machen. Die Anwendung oder Propagierung von Gewalt lehne sie strikt ab. Im Versuch einer Definition stellt Follen heraus, dass es sich weder um eine politische Partei, noch eine neue Sekte handelt. Die Erklärung setzt dazu an, Ziele zu konkretisieren und Gerüchten entgegenzuwirken.

Without objecting to any transient legal restraints and encouragements, which the influence of past servitude may render necessary, we claim for the colored man the immediate possession of personal independence and safety, the right to hold property, to be protected in all his family connections, to choose his own employment, to give valid testimony in any court of justice; we claim for him the free exercise of religion, the free expression of his sentiments, the use of every means of education by which he may fit himself as soon as possible for the exercise of every right enjoyed by the white man. This is what we mean by immediate abolition. (Follen 1842, V, 224)

Follen empfiehlt auch in den folgenden Jahren eine gewaltfreie Strategie, obwohl sich die Auseinandersetzungen zwischen Nord- und Südstaaten weiter zuspitzten. Das Arsenal zur Verfügung stehender Mittel sieht Follen in der freien Meinungsäußerung, dem Anschreiben gegen das bestehende Unrecht und der ungehinderten Publikation abolitionistischer Standpunkte. Veränderungen sollen mit Hilfe von Reformen und nicht mit kriegerischen Auseinandersetzungen bewirkt 
werden. Daher sein deutliches Fanal am Ende seiner Rede: "we aim at a national reform" (Follen 1842, I, 633).

\section{Zwischen gesellschaftlicher Integration und politischer Isolation}

Follens Aktionen innerhalb der Abolitionismusgesellschaften führten unter anderem dazu, dass die Anti-Sklaverei-Bewegung zunehmend als eine von Ausländern propagierte Rebellion gegen jenen Staat wahrgenommen wurde, der ihnen eine neue Existenz ermöglichte. Eine an Follen adressierte Kopie seiner Rede "Address to the People of the United States on the Subject of Slavery" vom Mai 1834, die ursprünglich an die Mitglieder des Kongresses und einflussreiche Persönlichkeiten in den Südstaaten gesandt wurde, enthielt diffamierende Anmerkungen. Follen solle sich seines Status' als Einwanderer bewusst werden und von Kritik an den Vereinigten Staaten gefälligst absehen. "A foreigner should recollect the protection afforded him by the institutions of this country, when he undertakes to cast a firebrand among the people, by which they may be destroyed" (Follen 1842, I, 629). Eliza Lee Cabot berichtet, wie eine Zeitung aus Boston die Gelegenheit nutzte, um seinen Ruf als ehrbaren Bürger in Frage zu stellen: "About this time a very gross attack was made upon him in one of the Boston papers; and, lest he should not himself know of it, the paper was sent to him, with the offensive paragraph marked for his particular notice" (Follen 1842, I, 343). Die Presse, die sich für die Belange der Sklavenhalter einsetzte, publizierte zunehmend Anklagen gegen sogenannte "foreign meddlers" - Ausländer also, die wagten, sich in inneramerikanische Belange einzumischen. Neben Follen gehörten hierzu die Engländer George Thompson und Harriet Martineau (Martineau 1877, II, 30). Vor allem Garrison setzte sich für die Reputation Follens ein und antwortete im Liberator 1834: "We wish we had more such foreigners among us." Damit zementierte er allerdings die Wahrnehmung Follens als Ausländer, anstatt die Argumentation der propagandistischen Presse unter Verweis auf seine amerikanische Staatsbürgerschaft zu entkräften. Trotz der großen Unterstützung für Follen von Persönlichkeiten wie Ellery Channing, Lydia Maria Child, Harriet Martineau und Theodore Parker zeigte die Reaktion seines Freundes und Mitstreiters Samuel May, dass die generelle Wahrnehmung von Follens Auftreten auch in den eigenen Reihen kaum von seiner Vergangenheit und ehemaligen Nationalität zu entkoppeln war. Die Anti-Slavery Society wählte Follen 1836 zum Sprecher in einer gerichtlichen Auseinandersetzung, in welcher der vom Gouverneur von Massachusetts erhobene Vorwurf des Gesetzesverstoßes entkräftet werden sollte. May hält die Vorgänge wie folgt fest:

His [Follen's] conduct on that memorable occasion commanded your admiration. It was worthy of himself. Standing before that committee, he evinced the same calm, invincible spirit of resistance to wrong, that had animated him when he withstood, at Basel, the demands of the Allied Sovereigns of Europe. In both cases it was principle, that he contended for. In both it was the violation of principle, that he chiefly dreaded. (Follen 1842, I, 402) 
Die scheinbare Wiederholung einer Auseinandersetzung zwischen Freiheitskämpfer und Despot auf amerikanischem Boden kennzeichnet Follen als den "besseren Amerikaner," der dem tyrannischen Staatsmann aus Massachusetts standhaft widerspricht. In ähnlicher Weise verklärt Martineau in ihrer Autobiographie die Gestalt Follens zum heldenhaften Patrioten, der auf beiden Kontinenten Opfer eines tyrannischen System wurde.

Dr. Follen, the patriot hero of Germany, the student, the poet, the philosopher, the victim of the Holy Alliance, the Christian teacher, the American abolitionist, and the victim of American despotism. [...] He was one of those rare great spirits that find no alternative at the call of a great cause but obedience. He was the only European exile of that vintage who declined to prosper as an American by flattering the nation's sin, - so rare is the virtue that can pour out of its life-blood twice. While suffering proscription from the land of his birth, he identified himself with Garrison among the earliest, and suffered, with the rest, a fresh proscription from the land of his love and his adoption. (Martineau 1877, II, 279)

Während May und Martineau die Prinzipientreue und moralische Geradlinigkeit im Denken Follens hervorzuheben versuchten, diffamierte die dem Abolitionismus gegenüber kritisch gestimmte Presse Follen als gescheiterten europäischen Flüchtling und amerikanischen Störenfried. In den Memoiren seiner Frau wird deutlich, dass Follen psychologisch unter dem Stigma des "Ausländers" litt (Follen 1842, I, 403). Doch seine Person bildete keine Ausnahme in der öffentlichen Verurteilung angesichts seiner abolitionistischen Tätigkeiten. Die 30er Jahre verzeichneten im Vergleich zu vorangehenden und nachfolgenden Dekaden die größte Anzahl kollektiver gewaltsamer Ausschreitungen im städtischen Milieu (Richards 1970, 23). Auch May und Garrison wurden Opfer von Diskriminierung und Diskreditierung. Letzterer musste sich 1829 einer Verleumdungsklage stellen; 1831 setzte der Staat Georgia ein Kopfgeld von $\$ 5000$ für Garrisons Gefangennahme aus; 1835 fiel Garrison fast einem Lynch-Mob in Boston zum Opfer (Mayer 1998, 200-10). Den Brennpunkt des öffentlichen Aufruhrs bildete die angekündigte Rede des Engländers George Thompson im Rahmen einer Veranstaltung der Boston Female Anti-Slavery Society. Auch er wurde bereits Wochen zuvor als ausländischer Agitator denunziert. Berichte warnten ihn davor, dass eine aufgebrachte Volksmenge sich gewalttätig gegen ihn erheben werde. Schließlich wurden $\$ 100$ Belohnung für denjenigen ausgeschrieben, der zuerst dem "Ausländer" martialisch die Leviten lese. Als Thompson am Tag der Kundgebung nicht anzutreffen war, übertrug sich der Hass auf Garrison, der wie ein gequältes Opfertier mit einem Strick um den Hals über den Marktplatz gezerrt und nur durch das beherzte Eingreifen eines Polizisten durch Inhaftierung dem Lynchmob entgehen konnte. Vor allem im Süden der Vereinigten Staaten kam es wiederholt zu Vorfällen, in denen ein aufgebrachter städtischer Mob Abolitionisten teerte, federte und anschließend vertrieb. Doch noch drastischere Fälle sind zu verzeichnen: Der abolitionistische Zeitungsherausgeber Elijah Lovejoy (18021837) wurde aufgrund seiner politischen Haltung 1837 in Alton, Illinois, ermor$\operatorname{det}$ (Mayer 1998, 237). 
Die Öffentlichkeit und rechtlichen Institutionen sanktionierten gewisse Formen der Gewalt als legitimes Mittel zum Ausdruck von Dissens, da sie keine reale Bedrohung für die Strukturen des gesellschaftlichen Zusammenlebens darstellten. Die Vorwürfe gegen die Abolitionisten konzentrierten sich auf sechs Aspekte: 1) Ablehnung der Konstitution als Grundlage der Staatengemeinschaft, 2) vorsätzliche Zerstörung des Wirtschaftssystems der Südstaaten, 3) Verschwörung mit Kaufleuten der Nordstaaten, 4) Befürwortung des Bürgerrechts für Afroamerikaner, 5) Toleranz von Mischehen ("racial amalgamation"), 6) Ablehnung sämtlicher Institutionen, die sich der Emanzipation verweigern.

Vor allem die örtlichen Intellektuellen oder wirtschaftlichen Eliten gehörten zu den Initiatoren der Aufstände in New York City, Utica, Philadelphia, Cincinnati oder Boston. Kennzeichnend für die Verharmlosung der in solchen Situationen eskalierenden Gewaltbereitschaft sind die Meldungen in den lokalen Zeitungen. Die am folgenden Tag in James Homers Commercial Gazette erschienene Meldung ist repräsentativ für den allgemeinen Tenor. Sie belegt darüber hinaus, dass es sich bei den Drahtziehern der Ausschreitungen vor allem um einflussreiche und wirtschaftlich erfolgreiche Bürger handelte. "[It was nothing but] a meeting of gentlemen of property and standing from all parts of the city" (Weinbaum 1979, 207). Dieser Vorfall verdeutlicht, daß sich der Haß der Bevölkerung nicht nur auf ausländische Aktivisten unter den Abolitionisten richtete, sondern ihre nationale Herkunft als ein willkommenes propagandistisches Mittel zur Meinungsbildung in den Medien fungierte. In ihren Aufzeichnungen zum Klima unter der amerikanischen Bevölkerung hält Martineau fest, dass Nichtamerikaner zwar gewalttätige Attacken zu fürchten hatten, dass sie sich allerdings nicht auf sie beschränkten.

George Thompson was then on sea, having narrowly escaped with his life; and the fury against "foreign incendiaries" ran high. Houses had been sacked; children had been carried through the snow from their beds at midnight: travellers had been lynched in the market-places, as well as in the woods; and there was no safety for any one, native or foreign [...]. (Martineau 1838, 30)

In einer Rede vom 20. Januar 1836 zum jährlichen Zusammentreffen der Massachusetts Anti-Slavery Society geht Follen explizit auf die Frage der Ausländer und die Intervention in der Sklavenfrage ein. Es ging ihm nicht um Beliebtheit und öffentliche Anerkennung. Denn mit der vorweg geschickten unpopulären Forderung, dass auch ehemalige Sklaven an den Treffen teilnehmen und der Gesellschaft beitreten müssten, demaskierte er Praktiken der Heuchelei selbst unter den Abolitionisten.

But, as for any meetings and associations designed for the establishment of human rights, - how can we have the effrontery to expect the white slave-holder of the South to live on terms of civil equality with his colored slave, if we, the white Abolitionists of the North, will not admit colored freemen as members of our anti-slavery societies? (Follen 1842, I, 629)

Follen spricht Tacheles. Er scheut sich nicht, auch in den eigenen Reihen die Borniertheit und Voreingenommenheit einiger Mitglieder der Anti-Slavery Society anzuprangern. Damit bestätigt er die scharfsinnige Beobachtung Tocquevilles, dass 
die Vorurteile im Norden der Vereinigten Staaten proportional zur tatsächlichen Emanzipation der Sklaven steigen (Tocqueville 2004, 329). Solche Denkmuster kommen auch in der Haltung gegenüber Ausländern zum Tragen, denn Follen ruft angesichts der anhaltenden Diffamierungen zum Umdenken auf: "I assert, that with regard to this cause, foreigners and citizens have the same duties and the same rights" (Follen 1842, I, 629). Aus beiden Statements erwächst der Eindruck, dass die Forderung nach Bürgerrechten für Unfreie und die Anerkennung Follens als amerikanischer Staatsbürger miteinander gekoppelt sind. Da er zu diesem Zeitpunkt die amerikanische Staatsbürgerschaft bereits seit sechs Jahren besaß und sich als Harvardprofessor verdient gemacht hatte, musste ihn der Vorwurf des "ausländischen Aufrührers" besonders hart treffen. An diesem Umstand entzündet sich ein Kernproblem der amerikanischen Widersprüche zwischen theoretischem Anspruch und einer genau entgegengesetzten Wirklichkeit: "I should have passed over, in silence, these petty vexations, as solitary exceptions to the uniform experience of generous confidence and kindness, which I have never ceased to enjoy in this community, if it were not for the great principle involved in these disagreeable trifles" (Follen 1842, I, 629). Follen erzählt das Gleichnis vom barmherzigen Samariter, um es auf die Behandlung der Sklaven umzumünzen. An die Stelle des eingebildeten Pharisäers, der einem von Räubern überfallenen Menschen keine Hilfe leistet, treten stellvertretend Priester und weltliche Machthaber. Wenn Ausländer sich menschenfreundlich zeigen, sollten gerade amerikanische Staatsbürger, die für sich eine moralische Vorbildfunktion in Anspruch nehmen, nicht ausrufen: "He is a foreigner; an English emissary; mob him! Tar and feather him!" (Follen 1842, I, 630). Der wahre Ausländer sei nämlich derjenige, der sich einem Hilfsbedürftigen verschließe und die Menschenrechte nur selektiv anerkenne.

\section{Bilanz}

Follens Protest und politisches Engagement nimmt eine Sonderstellung unter deutschen Einwanderern und Reisenden ein, die in der Zeit vor 1848 über die Realitäten der politischen Strukturen in den USA schrieben. Ihre Bemerkungen zur Sklaverei bleiben oftmals oberflächlich und unreflektiert, wie die Konversionsgeschichten eines Ernst Braun (Ideen über die Auswanderung nach Amerika, 1827), eines Paul Wilhelm von Württemberg (Reise nach dem nördlichen Amerika, 1835) oder einer Clara Gerstner (Beschreibung einer Reise, 1842) zeigen. Der anfangs empfundenen Abscheu gegenüber der Sklaverei weicht in den Reiseberichten die Erkenntnis, dass die Sklaven in guten Arbeitsverhältnissen stünden, dass die wirtschaftlichen Umstände einen solchen Dienst bedingten und die Sklaverei somit nicht mehr zu beseitigen sei. Peter Brenner argumentiert, dass der freundschaftliche Kontakt deutscher Reisender mit Plantagenbesitzern eine Gleichsetzung in der sozialen Ordnung implizierte (Brenner 1991, 372). Die Aufwertung des sozialen Selbstwertgefühls der deutschen Amerikabesucher führte somit zu einer Neubewertung der Sklaverei, die weniger von intellektueller Durchdringung des Problems als von Selbstgefälligkeit geprägt war. Follens emphatische Hinwendung zu 
den rechtlich benachteiligten Sklaven beruht nicht auf einer widerwillig akzeptierten Identifikation mit den Schwächsten im amerikanischen Gemeinwesen. Sie zeugt von einem Gerechtigkeitssinn, den Follen als Burschenschaftler in Giessen ausbildete. Die propagierten Mittel zur Durchsetzung wandelten sich jedoch nachhaltig vom Aufruf zum gewaltsamen Umsturz zum moralisch-rechtlichen Einsatz für Reformen. Die Fremdheitserfahrung, öffentliche Diffamierung und soziale Abstufung findet bei Follen im Vergleich zu den meisten Einwanderern zeitlich entgegengesetzt statt. Denn erst nach seinem offiziellen Eintritt in die AntiSlavery Society beginnt seine Persönlichkeit und Herkunft die Öffentlichkeit zu polarisieren. Auch die gesellschaftliche Aufwertung der Frauen, für die sich Follen im Rahmen seiner Tätigkeit als Abolitionist einsetzt, unterscheidet ihn von einem in der Sekundärliteratur betonten Hierarchisierungsdenken unter deutschen Immigranten. Wynfried Kriegleders Darlegungen zur Frage, inwiefern sich in dem Rollenverständnis der Immigranten zu den Afroamerikanern das deutsche Patriarchat manifestiert, werden mit Follens Wirken durchbrochen. Denn der Deutsch-Amerikaner entwickelt ein außergewöhnliches Sensorium für die Widersprüche der amerikanischen Ideale auf der einen Seite und der gesellschaftlichen Praxis gegenüber ethnischen Minderheiten und Frauen auf der anderen Seite. Anstatt eine Aufwertung der deutschen Kultur und seiner Vergangenheit in Relation zu der afroamerikanischen Bevölkerung voranzutreiben, widmet er sich der Aufgabe, die rechtliche Gleichstellung aller Menschen auf dem nordamerikanischen Kontinent $\mathrm{zu}$ verwirklichen. Sezessionistischen Tendenzen und der Befürwortung von Gewalt, wie sie beispielsweise William Lloyd Garrison forderte, hat Follen zu keinem Zeitpunkt gehuldigt. Seine Bemühungen, zu einem vorbildlichen Repräsentanten demokratischer Grundsätze in Amerika zu avancieren, scheitern indes in dem Moment, als Parallelen zwischen seiner Funktion als Literaturprofessor und seiner Rolle als Abolitionist entstehen.

Mit seiner Sprachkompetenz, der neu erworbenen Staatsbürgerschaft und einer beruflichen success story positionierte sich Follen als überzeugter Amerikaner. Das öffentliche Bild bleibt jedoch von seiner geschichtlichen Vergangenheit und kulturellen Prägung in Deutschland bestimmt. Die Wahrnehmung von Follens Dissens läßt sich nicht von dem europäischen Teil seiner Biographie entkoppeln. Entgegen der Erfahrung von rechtlichen Missständen und öffentlichen Diffamierungen als "ausländischer Brandstifter" schlägt seine ursprüngliche Amerikabegeisterung nicht in die desillusionierte "Amerikamüdigkeit" eines Nikolaus Lenau, Dietrich von Bülow oder Ferdinand Kürnberger um. Stattdessen offenbart sein selbstloser Einsatz einen bis dato verkannten "Verteidiger der Freiheit und Freund menschlicher Würde" - ein Titel, den im New Yorker Morningside Park bisher lediglich das Denkmal des Deutsch-Amerikaners Carl Schurz ziert.

\section{Literaturverzeichnis}

Brenner, Peter (1991). Reisen in die Neue Welt: Die Erfabrung Nordamerikas in deutschen Reise- und Auswanderungsberichten des 19. Jahrbunderts. Tübingen: Niemeyer. 
Braun, Ernst (1827). Ideen über die Auswanderung nach Amerika. Göttingen.

Brumm, Ursula (1995). "Charles Follen: Kultureller Mittler bei den amerikanischen Transzendentalisten." Udo J. Hebel und Karl Ortseifen, eds. Transatlantic Encounter: Studies in European-American Relations. Trier: Wissenschaftlicher Verlag, 146-56.

Buell, Lawrence (1993). New England Literary Culture: From Revolution through Renaissance. Cambridge: Cambridge University Press.

Depkat, Volker (1998). Amerikabilder in politischen Diskursen: Deutsche Zeitschriften von 1789 bis 1830 . Stuttgart: Klett-Cotta.

Douglass, Frederick (1893). "What to the Slave is the 4th of July?" James M. Gregory, ed. Frederick Douglass, the Orator. Springfield, MA: Willey and Co., 103-6.

Dubois, Ellen (1978). "Women's Rights and Abolition: The Nature of the Connection." Feminism and Suffrage: The Emergence of an Independent Women's Movement in America, 1848-1869. Ithaca, NY: Cornell University Press.

Emerson, Ralph Waldo (1939). The Letters of Ralph Waldo Emerson. Ed. Ralph L. Rusk. 6 vols. New York: Columbia University Press.

- (1979). "Man the Reformer." Alfred R. Ferguson and Robert E. Spiller, eds. Nature, Addresses and Lectures. Cambridge: Belknap Press of Harvard University Press, 141-60.

Faust, Albert Bernhardt (1927). The German Element in the United States. 2 vols. New York: The Steuben Society of America.

Fluck, Winfried (2002). "The Man Who Became Weary of America: Ferdinand Kürnberger's Novel Der Amerika-Müde (1855).” Winfried Fluck and Werner Sollors, eds. German? American? Literature? New Directions in German-American Studies. New York et al.: Peter Lang, 171-206.

Follen, Charles (1842). The Works of Charles Follen with a Memoir of his Life. Ed. Eliza Lee Cabot. 5 vols. Boston: Hilliard, Gray and Company.

Garrison, William Lloyd (1852). "An Address to the American Colonization Society." Selections from the Writings and Speeches of William Lloyd Garrison, with an Appendix. Boston: R.F. Walcut, 45-61.

- (2000). "Declaration of the National Antislavery Convention (1833)." Mason Lowance, ed. Against Slavery: An Abolitionist Reader. New York: Penguin Books, 119-22.

Gerstner, Clara (1842). Beschreibung einer Reise durch die Vereinigten Staaten von Nordamerika in den Jabren 1838 bis 1840. Leipzig.

Gougeon, Len and Joel Myerson, eds. (1995). Emerson's Antislavery Writings. New Haven and London: Yale University Press.

Hardtwig, Wolfgang (1985). Vormärz: Der monarchische Staat und das Bürgertum. München: Deutscher Taschenbuchverlag.

Haupt, Hermann (1907). Karl Follen und die Gießener Schwarzen. Gießen: Töpelmann.

Heubner, Theodore (1962). The Germans in America. Philadelphia: Chilton. 
Körner, Gustav Philipp (1880). Das deutsche Element in den Vereinigten Staaten 1818-1848. Cincinnati: Wilde.

Körner, Theodor (1910). Theodor Körner: Briefwechsel mit den Seinen. Ed. Augusta Weldler-Steinberg. Leipzig: Quelle \& Meyer.

Kriegleder, Wynfried (1998). Vorwärts in die Vergangenheit: Das Bild der USA im deutschsprachigen Roman von 1776 bis 1855. Tübingen: Stauffenburg.

Luys, Karin (1992). Die Anfänge der deutschen Nationalbewegung von 1815-1919. Münster: Nodus.

Martineau, Harriet (1838). Martyr Age of the United States. Boston: Weeks, Jordan and Co.

- (1877). Autobiography. 3 vols. London: Smith, Elder.

Mayer, Henry (1998). All on Fire: William Lloyd Garrison and the Abolition of Slavery. New York: St. Martin's Griffin.

Mehring, Frank (2004). “'American German Mania': Kritische Anmerkungen zur Goethe-Kontroverse aus deutsch-amerikanischer Perspektive 1815-1850." Goethe im Vormärz. Forum Vormärz Forschung Jahrbuch 2003. Bielefeld: Aisthesis, 171-202.

Mitchell, Harvey (2002). America after Tocqueville: Democracy against Difference. Cambridge: Cambridge University Press.

Moraw, Peter (1990). Kleine Geschichte der Universität Giessen. Giessen: Verlag der Ferber'schen Universitäts-Buchhandlung Giessen.

Münch, Friedrich (1873). Erinnerungen aus Deutschlands trübster Zeit. St. Louis: C. Witter.

Pochmann, Henry (1957). A German Culture in America: Philsophical and Literary Influences, 1600-1900. Madison, WI: University of Wisconsin Press.

Richards, Leonard L. (1970). "Gentlemen of Property and Standing:" Anti-Abolition Mobs in Jacksonian America. New York: Oxford University Press.

Sammons, Jeffrey L. (1998). Ideology, Mimesis, Fantasy: Charles Sealsfield, Friedrich Gerstäcker, Karl May, and Other German Novelists of America. Chapel Hill and London: The University of North Carolina Press.

Shore, Elliott (2001). "The German Part of American History.” Frank Trommler und Elliott Shore, eds. The German-American Encounter: Conflict and Cooperation between Two Cultures, 1800-2000. New York and Oxford: Berghahn Books, 3-6.

Spevack, Edmund (1997). Charles Follen's Search for Nationality and Freedom: Germany and America 1796-1840. Cambridge, MA: Harvard University Press.

Spindler, George Washington (1917). Karl Follen: A Biographical Study. Chicago: Chicago University Press.

Stange, Douglas C. (1977). Patterns of Antislavery Among American Humanitarians. Rutherford, NJ: Fairleigh Dickinson Univeristy Press.

Tocqueville, Alexis de (2004). Democracy in America. Trans., ed. with an introduction by Harvey C. Mansfield and Delba Winthrop. Chicago and London: University of Chicago Press. 
Totten, Christine M. (1964). Deutschland - Soll und Haben: Amerikas Deutschlandbild. München: Rütten und Loening.

Tolzmann, Don Heinrich (2000). The German-American Experience. New York: Humanity Books.

Treitschke, Heinrich von (1927). Deutsche Geschichte im 19. Jabrbundert. 3 vols. Leipzig: Hirzel.

Vogel, Stanley M. (1917). German Literary Influences on the American Transcendentalists. New Haven, CT: Yale University Press.

Weinbaum, Paul O. (1979). Mobs and Demagognes: The New York Response to Collective Violence in the Early Nineteenth Century. Ann Arbor, MI: University of Michigan International Research Press.

Württemberg, Paul Wilhelm von (1835). Reise nach dem nördlichen Amerika in den Jabren 1822-24. Stuttgart.

Wüst, Ebenso Julia (1936). "Karl Follen: Seine Ideenwelt und ihre Wirklichkeit.” Mitteilungen des Oberhessischen Geschichtsvereins 33, 5-139. 